\title{
Diagnostic Value of Plain Abdominal Radiography in Stroke Patients With Bowel Dysfunction
}

\author{
Hyo Jeong Moon, MD, Se Eung Noh, MD, Ji Hee Kim, MD, Min Cheol Joo, MD \\ Department of Rehabilitation Medicine and Institute of Wonkwang Medical Science, \\ Wonkwang University School of Medicine, Iksan, Korea
}

Objective To evaluate the diagnostic value of plain abdominal radiography in stroke patients with bowel dysfunction.

Methods A total of 59 stroke patients were recruited and assigned into constipation or non-constipation group. Patients were interviewed to obtain clinical information, constipation score, and Bristol stool form scale. The total and segmental colon transit time (CTT) was measured using radio-opaque markers (Kolomark). The degree of stool retention was evaluated by plain abdominal radiography and scored by two different methods (Starreveld score and Leech score). The relationship between the clinical aspects, CTT, and stool retention score using plain abdominal radiography was determined.

Results Average constipation score was $4.59 \pm 2.16$. Average Bristol stool form scale was $3.86 \pm 1.13$. The total and segmental CTTs showed significant differences between the constipation and non-constipation groups. There was statistically significant $(\mathrm{p}<0.05)$ correlation between the total CTT and constipation score or between Starreveld score and Leech score. Each segmental CTT showed significant correlation $(\mathrm{p}<0.05)$ between segmental stool retention scores.

Conclusion The stool retention score showed significant correlation with constipation score as well as total and segmental CTT. Thus, plain abdominal radiography is a simple and convenient method for the evaluation of bowel dysfunction in stroke patients.

Keywords Constipation, Stroke, Abdominal radiography, Colon transit time

Received July 23, 2014; Accepted September 23, 2014

Corresponding author: Min Cheol Joo

Department of Rehabilitation Medicine and Institute of Wonkwang Medical Science, Wonkwang University School of Medicine, 895 Muwang-ro, Iksan 570-974, Korea

Tel: +82-63-859-1612, Fax: +82-63-843-1385, E-mail: mcjoo68@wku.ac.kr

(c) This is an open-access article distributed under the terms of the Creative Commons Attribution Non-Commercial License (http://creativecommons. org/licenses/by-nc/3.0) which permits unrestricted noncommercial use, distribution, and reproduction in any medium, provided the original work is properly cited.

Copyright $\odot 2015$ by Korean Academy of Rehabilitation Medicine

\section{INTRODUCTION}

Post-stroke bowel dysfunction, one of the most common complications observed in $30 \%$ to $60 \%$ of stroke patients [1-3], usually manifests as constipation, fecal incontinence, and fecal impaction. Post-stroke bowel dysfunction causes social and psychological retreat, making it difficult for patients to perform independent activities of daily living or participate in social life. Despite its incidence and severity, the importance of gastrointestinal 
problems in stroke patients is overlooked. There are few reports on the evaluation of bowel movement function.

Previous studies on constipation in stroke patients have relied on subjective symptoms, such as the frequency of defecation for diagnosis. This is why constipation is considered as a symptom based disorder. However, it has been reported [4] that patients do not have a clear memory of bowel habits. Therefore, it is necessary to have objective examination methods, such as plain abdominal radiography and colon transit time (CTT), to provide appropriate bowel management and treatment guidelines. Yi et al. [5] and Lim et al. [6] have reported that there is significant statistical difference in constipation score and CTT between constipation group and non-constipation group of stroke patients, showing that CTT is an objective method for the evaluation of motility of the colon. However, CTT has some limitations in its applicability for patients with swallowing difficulty. In addition, CTT requires twice the time it takes to do plain abdominal radiography which takes at least 4 days to complete the entire process to obtain examination results. Leech et al. [7] reported that plain abdominal radiography showed high reproducibility and high inter-investigator consistency in evaluating stool retention among children with constipation. Starreveld et al. [8] also reported that plain abdominal radiography was significantly correlated with characteristics of the bowel pattern, such as bowel frequency, hardness, and weight of the stools among adult patients with constipation. Recently, the diagnostic accuracy of plain abdominal radiography between subjective symptoms of constipation is found to be very poor for stroke patients. Those previous studies using the plain abdominal radiography have focused on children or functional constipation in adult patients. They observed that the correlations with subjective symptoms had the limitation of having no comparison with the CTT. The pattern of bowel dysfunction in stroke patients may be different from the pattern of bowel dysfunction in populations that have functional constipations. No previous study on stroke patients has determined whether plain abdominal radiography could evaluate the degree of stool retention quantitatively or whether plain abdominal radiography results are helpful in guiding proper management. Compared to CTT, plain abdominal radiography has advantages in its simple examination process, reduced costs, and less radiation exposure. Whether plain abdominal radiography will be helpful in creating appropriate treatment guidelines by evaluating the distribution of stools in the colon, appropriate bowel management, and the degree of stool retention was unclear. Therefore, the objective of this study was to evaluate the relationship between the degree of stool retention and bowel pattern using plain abdominal radiography and CTT in stroke patients. The diagnostic value of plain abdominal radiography for its convenience in clinical use for proper bowel management was also determined in this study.

\section{MATERIALS AND METHODS}

\section{Subjects}

Stroke patients who were admitted to the rehabilitation medicine department from January 2009 to February 2012 and diagnosed with hemorrhagic stroke or ischemic stroke completed a questionnaire, including constipation score, and morphological stool characteristics using Bristol stool form scale on their bowel pattern. With the use of plain abdominal radiography, stool retention score was evaluated. A total of 59 subjects who also proceeded with CTT were included in this study. Those who had a history of gastrointestinal disorder or history of gastrointestinal surgery were excluded. All subjects maintained ordinary life styles. They were on medication during evaluation.

\section{Methods}

\section{Evaluation of bowel pattern}

With respect to bowel pattern, the constipation score was evaluated using the Rome II Diagnostic Criteria [9]. The Bristol stool form scales were recorded. For Rome II Diagnostic Criteria, bowel frequency for 7 days with the presence of straining, lumpy or hard stools, sensation of incomplete evacuation or anorectal obstruction, and the need for additional manual maneuvers to facilitate defecation were recorded and scored [10]. For bowel frequency, 0 point was scored for ' 3 or more bowel movements per week,' 1 point for ' $1-2$ per week,' 2 points for ' 1 for 10 days,' and 3 points for 'hard to move the bowel for more than 10 days.' For items of 'straining,' 'lumpy or hard stools,' 'sensation of incomplete evacuation,' ‘sensation of anorectal obstruction' and 'need for additional manual maneuvers to facilitate defecation,' 0 point was assigned for 'none,' 1 point for 'at least 1 among 4 times,' 2 points for ' $2-3$ times among 4 times', and 3 points for 
'always.' The range of the constipation score was between 0 and 18, with higher score indicating more severe constipation. To examine stool characteristics morphologically, the form of the stool after a bowel movement was checked on the Bristol stool form scale which classified the form into 7 types (from type 1 to type 7 ) [11] according to the degree of the form and hardness.

\section{Colon transit time}

Total and segmental CTT was evaluated to measure colonic motility. The subject was administered one capsule of Kolomark (M.I.Tech, Seoul, Korea) containing 20 radio-opaque markers for 3 days at 9:00 AM. A plain abdominal radiography was taken at same time on the 4 th day.

To measure the total and segmental CTT, the total colon was divided into the right colon, left colon, and rectosigmoid colon. The total and segmental CTT times were calculated using the method reported by Arhan et al. [12].

\section{Plain abdominal radiography}

Plain abdominal radiography on the 4th day was used for evaluation of CTT. Two well-trained radiologists blinded to patient information analyzed the plain abdominal radiography using the Starreveld score and Leech score to determine the degree of stool retention. The Starreveld score was based on the entire colon divided into 4 segments, i.e., the ascending, transverse, descending, and rectosigmoid colon. Leech score was based on the entire colon divided into 3 segments, i.e., the right, left, and rectosigmoid colon. Both scoring systems assigned 0 point if no stool was ever observed, 1 point if a small amount of stool was observed, 2 points if a moderate amount of stool was observed, and 3 points if stool was seriously delayed. The Starreveld score was recorded on a scale of 0 to12 points, whereas the Leech score was recorded on a scale of 0 to 9 points.

\section{Statistical analysis}

Pearson correlation coefficients were used to analyze correlations in the bowel patterns, including the constipation score and the Bristol stool form scale, the degree of stool retention on plain abdominal radiography and CTT, as well as inter-investigator reliability. Between the constipation and non-constipation groups, the differences in constipation scores, CTT, and the degree of stool retention were evaluated by independent-test using a significance level of $\mathrm{p}<0.05$. Statistical analysis was performed using SPSS ver. 19.4 (IBM SPSS, Armonk, NY, USA).

\section{RESULTS}

\section{General characteristics of the subjects}

A total of 39 male patients and 20 female patients with ages ranging from 21 to 89 years were included in the study. The average age was $59.59 \pm 13.91$ years. Thirty-four subjects had ischemic stroke and 25 had hemorrhagic stroke. The average duration from the stroke to the examination was $10.23 \pm 16.33$ months. Modified Barthel Index of the subjects ranged from 7 to 100 , with an average of $62.38 \pm 25.56$. The Korean version of Mini-Mental State Examination ranged from 0 to 30, with an average of $22.03 \pm 7.44$ (Table 1).

\section{Evaluation of the bowel pattern}

Of the 59 patients, 45 were assigned to the constipation group. The remaining 14 patients were assigned to the non-constipation group based on the Rome II Diagnostic Criteria. Their constipation scores ranged from 1 to 13 , with an average score of $4.58 \pm 2.16$. Constipation and non-constipation groups scored $5.11 \pm 2.11$ and $2.92 \pm 1.38$, respectively, with a significantly higher constipation score in the constipation group (Fig. 1A). The Bristol stool form scale ranged from 1 to 6 , with an average score of 3.86 \pm 1.13 . Constipation and non-constipation groups

Table 1. General characteristics of stroke patients $(n=59)$

\begin{tabular}{|cc|}
\hline \multicolumn{1}{|c}{ Demographic factor } & Value \\
\hline Age (yr) & $59.59 \pm 13.91$ \\
\hline Sex (male:female) & $39: 20$ \\
\hline Duration of brain injury (mo) & $10.23 \pm 16.33$ \\
\hline Type of brain injury & \\
\hline Infarction & 34 \\
\hline Hemorrhage & 25 \\
\hline MBI & $62.38 \pm 25.56$ \\
\hline K-MMSE & $22.03 \pm 7.44$ \\
\hline
\end{tabular}

Values are presented as mean \pm standard deviation or number.

MBI, Modified Barthel Index; K-MMSE, Korean version of Mini-Mental State Examination. 
scored $3.68 \pm 1.18$ and $4.42 \pm 0.75$, respectively, with a significantly higher Bristol stool form score in the constipation group (Fig. 1B).

\section{Colon transit time}

CTT for the right colon, the left colon, and the rec- tosigmoid colon were $10.67 \pm 9.99,14.01 \pm 12.58$, and $11.47 \pm 12.02$ hours, respectively. The total CTT was $36.15 \pm 22.15$ hours, which was longer compared to the total and segmental CTT of normal people reported in previous studies [13]. The total CTT for both the constipation and the non-constipation groups were $40.76 \pm 22.36$ and
(A)

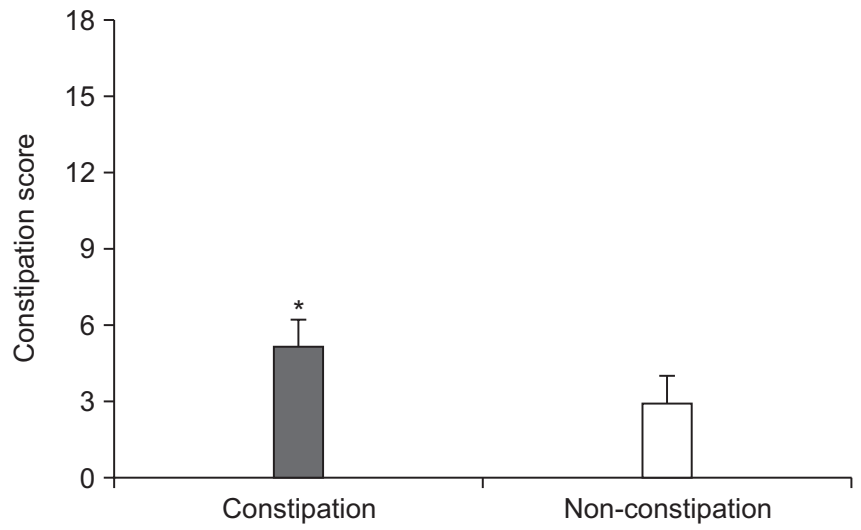

(B)

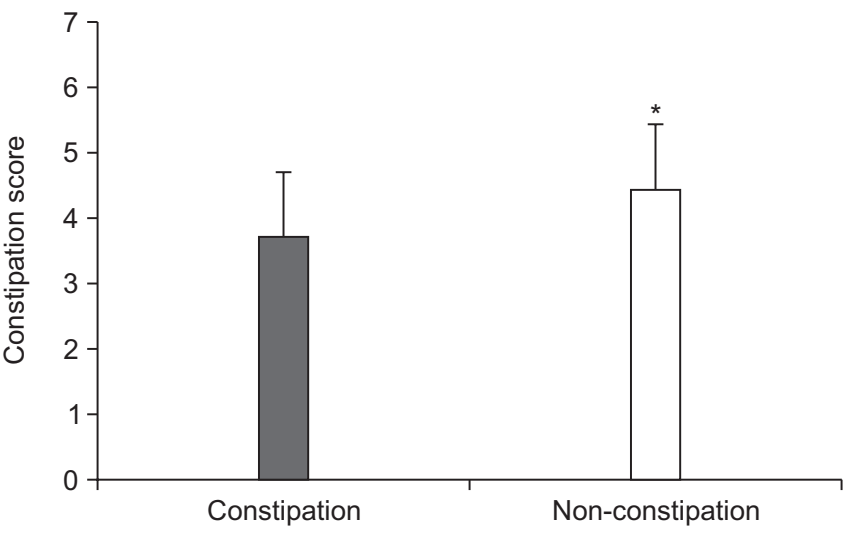

Fig. 1. The comparison of constipation score and Bristol stool form scale in the constipation and the non-constipation groups. Values were presented as mean \pm standard deviation. ${ }^{*} \mathrm{p}<0.05$, significant difference between the constipation and the non-constipation groups.

Table 2. The results of colon transit time between the constipation and the non-constipation groups

\begin{tabular}{lcccc}
\hline & Total & Right colon & Left colon & Rectosigmoid colon \\
\hline Constipation group & $40.76 \pm 22.36^{*}$ & $12.26 \pm 10.59$ & $16.74 \pm 13.08^{*}$ & $13.56 \pm 13.36^{*}$ \\
Non-constipation group & $27.17 \pm 14.70$ & $9.08 \pm 7.57$ & $10.02 \pm 8.90$ & $8.14 \pm 6.40$ \\
\hline
\end{tabular}

Values are presented as mean \pm standard deviation or number.

${ }^{*} \mathrm{p}<0.05$, significant difference between the constipation and the non-constipation groups.

(A)

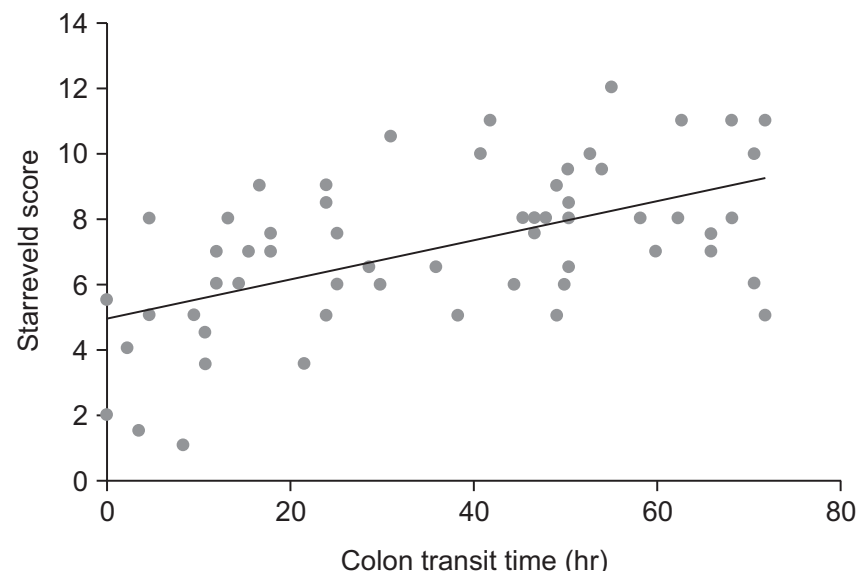

(B)

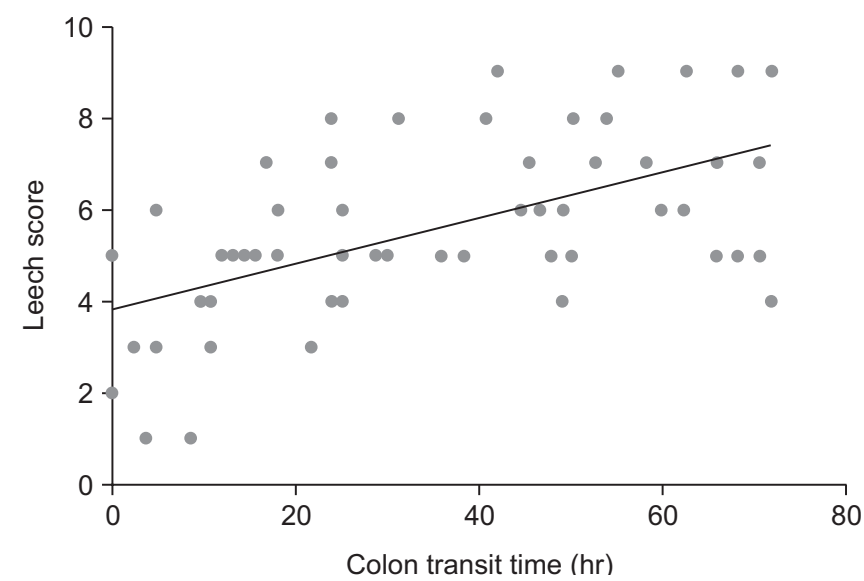

Fig. 2. Starreveld score (A) and Leech score (B) on each segment were evaluated by plain abdominal radiography. 
$27.17 \pm 14.70$ hours, respectively, with a statistically significant difference $(\mathrm{p}<0.05)$ between the two groups. With respect to the segmental CTT for the constipation and non-constipation groups, the right colon was $12.26 \pm 10.56$ and 9.08 \pm 7.57 hours, respectively. The left colon was $16.74 \pm 13.08$ and $10.02 \pm 8.90$ hours, respectively. The rectosigmoid colon was $13.56 \pm 13.36$ and $8.14 \pm 6.40$ hours, respectively. The segmental CTT for the left and rectosigmoid colons in the constipation group were significantly $(\mathrm{p}<0.05)$ higher than those in the non-constipation group (Table 2).

\section{Plain abdominal radiography}

Based on plain abdominal radiography, the Starreveld scores for the ascending, transverse, descending, and rectosigmoid colons were $2.26 \pm 0.77,1.34 \pm 0.76,1.89 \pm 0.79$, and 1.59 \pm 1.07 , respectively. The Starreveld score for the total colon was 7.11 \pm 2.39 . The Leech scores for the right, left, and rectosigmoid colons were $2.15 \pm 0.72,1.86 \pm 0.66$, and $1.54 \pm 0.99$, respectively. It was measured as $5.66 \pm 1.93$ at the total (Fig. 2). In the constipation and the nonconstipation groups, the Starreveld scores for the ascending colon were $2.22 \pm 0.82$ and $2.39 \pm 0.59$, respectively. The Starreveld scores for the transverse colon were $1.42 \pm 0.82$ and $1.14 \pm 0.49$, respectively. The Starreveld score at the descending colon in the constipation group was significantly $(\mathrm{p}<0.05)$ higher than that in the non-constipation group (1.87 \pm 0.86 vs. $1.96 \pm 0.49)$ There was no significant difference between the two groups in the total and rectosigmoid colon (Table 3). Regarding the constipation

(A)

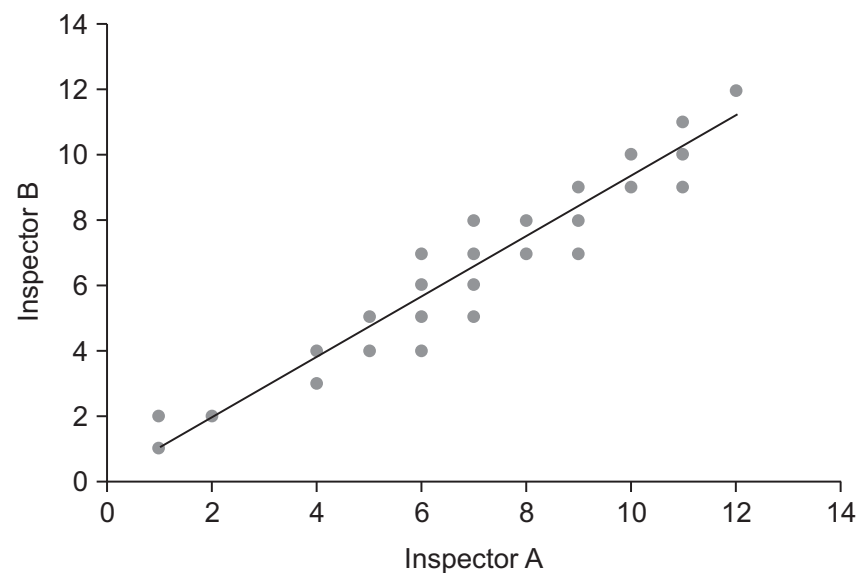

and non-constipation groups, the Leech scores at the right colon were $2.18 \pm 0.79$ and $2.03 \pm 0.41$, respectively. The Leech scores at the left colon were $1.86 \pm 0.74$ and $1.85 \pm 0.36$, with a significantly $(\mathrm{p}<0.05)$ higher score in the constipation group. However, there was no significant difference between the two groups in the total or the rectosigmoid colon (Table 3 ).

Table 3. The results of Starreveld and Leech scores between the constipation and the non-constipation group

\begin{tabular}{lcc}
\hline & $\begin{array}{c}\text { Constipation } \\
\text { group }\end{array}$ & $\begin{array}{c}\text { Non-constipation } \\
\text { group }\end{array}$ \\
\hline Starreveld score & & \\
\hline Ascending colon & $2.22 \pm 0.82$ & $2.39 \pm 0.59^{*}$ \\
Transverse colon & $1.42 \pm 0.82^{*}$ & $1.14 \pm 0.49$ \\
Descending colon & $1.87 \pm 0.86^{*}$ & $1.96 \pm 0.49$ \\
Rectosigmoid colon & $1.60 \pm 1.10$ & $1.57 \pm 1.01$ \\
\hline Total & $7.12 \pm 2.61$ & $7.07 \pm 1.56$ \\
Leech score & & \\
Right colon & $2.18 \pm 0.79^{*}$ & $2.03 \pm 0.41$ \\
\hline Left colon & $1.86 \pm 0.74^{*}$ & $1.85 \pm 0.36$ \\
\hline Rectosigmoid colon & $1.55 \pm 1.02$ & $1.50 \pm 0.91$ \\
\hline Total & $5.71 \pm 2.08$ & $5.50 \pm 1.40$ \\
\hline
\end{tabular}

Values are presented as mean \pm standard deviation or number.

${ }^{*} \mathrm{p}<0.05$, significant difference between the constipation and the non-constipation groups.
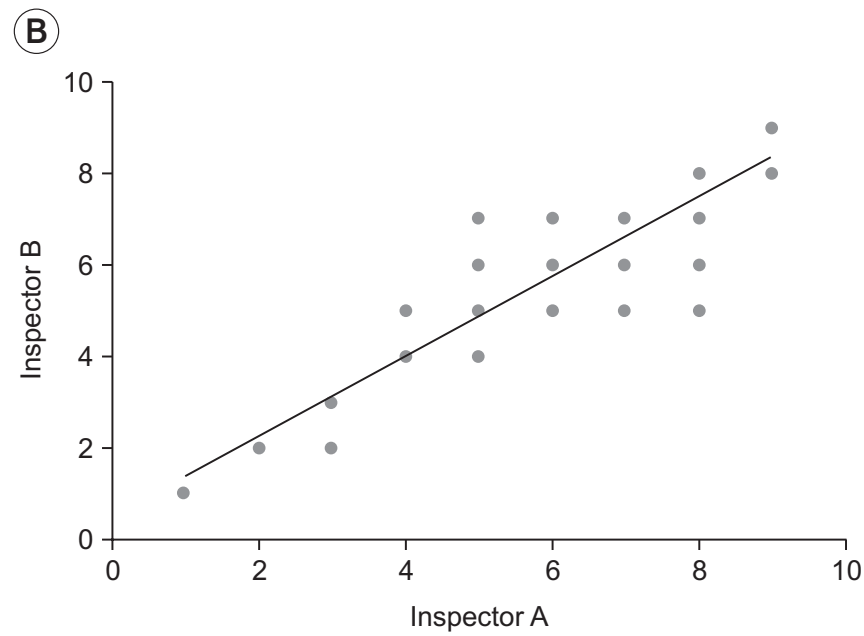

Fig. 3. The inter-inspector reliability on Starreveld score (A) and Leech score (B) was represented by scatter diagrams. There was a statistically significant correlation between the interpretations of inspectors $(r=0.953, r=0.905)$. 
Table 4. Correlation between segmental CTT and segmental Starreveld score

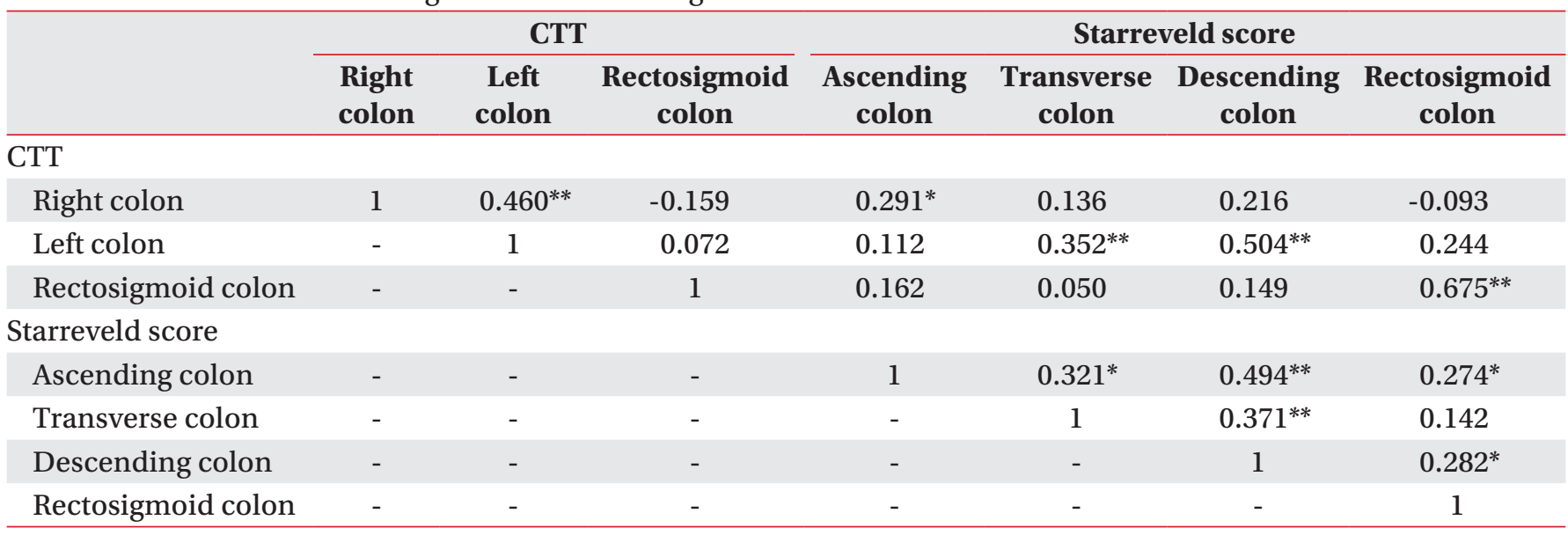

CTT, colon transit time.

${ }^{*} \mathrm{p}<0.05,{ }^{* *} \mathrm{p}<0.01$ by Pearson correlation analysis.

Table 5. Correlation between segmental CTT and segmental Leech score

\begin{tabular}{|c|c|c|c|c|c|c|}
\hline & \multicolumn{3}{|c|}{ CTT } & \multicolumn{3}{|c|}{ Leech score } \\
\hline & Right colon & Left colon & $\begin{array}{c}\text { Rectosigmoid } \\
\text { colon }\end{array}$ & Right colon & Left colon & $\begin{array}{c}\text { Rectosigmoid } \\
\text { colon }\end{array}$ \\
\hline Right colon & 1 & $0.460^{* *}$ & -0.159 & $0.394^{* *}$ & 0.128 & -0.072 \\
\hline Left colon & - & 1 & 0.072 & $0.258^{*}$ & $0.512^{* *}$ & 0.234 \\
\hline Right colon & - & - & - & 1 & $0.502^{* *}$ & $0.304^{*}$ \\
\hline Left colon & - & - & - & - & 1 & $0.333^{* *}$ \\
\hline Rectosigmoid colon & - & - & - & - & - & 1 \\
\hline
\end{tabular}

CTT, colon transit time.

${ }^{*} \mathrm{p}<0.05,{ }^{* *} \mathrm{p}<0.01$ by Pearson correlation analysis.

Inter-inspector reliability in reading plain abdominal radiography

The comparison of the consistency of the degree of stool retention on plain abdominal radiography evaluated by Starreveld score and Leech score showed $r=0.953$ and $\mathrm{r}=0.905$, indicating that the inter-investigator consistency was significantly high ( $p<0.01)$ (Fig. 3$)$.

Correlation between colon transit time and plain abdominal radiography

Correlation between segmental CTT and the Starreveld score were as follows: as the right CTT increased, the stool retention score at the ascending colon also increased; as the left CTT increased, the stool retention score at the transverse and descending colon also increased; as the rectosigmoid CTT increased, the stool retention score at the rectum also increased $(\mathrm{p}<0.05)$ (Table 4$)$.

Correlation between segmental CTT and the Leech score were as follows: as the right CTT increased, the stool retention score at the right colon also increased; as the left CTT increased, the stool retention score at the left colon also increased; as the rectosigmoid CTT increased, the stool retention score at the rectosigmoid score also increased $(\mathrm{p}<0.01)$ (Table 5).

As the total CTT increased, the score of the total colon for both the Starreveld score $(\mathrm{p}<0.05)$ and Leech score $(p<0.01)$ significantly increased, showing a significant positive correlation (Fig. 4). 
(A)

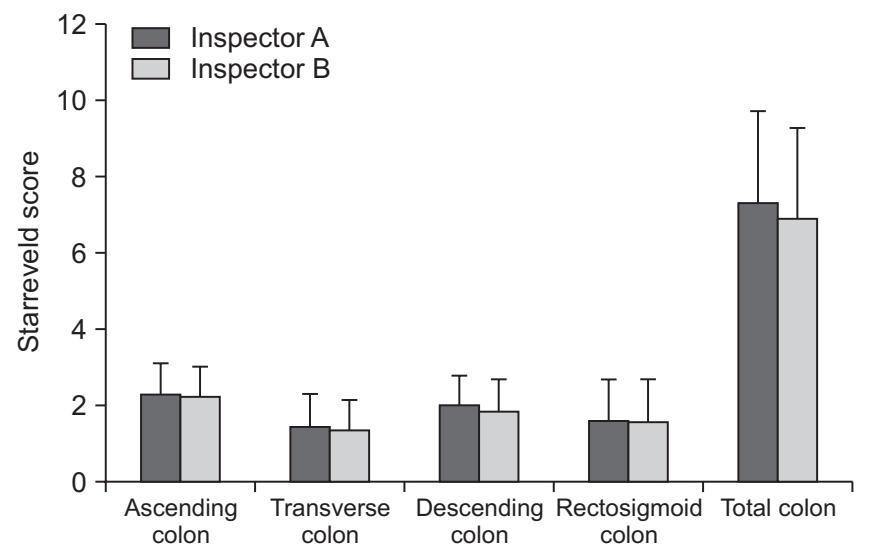

(B)

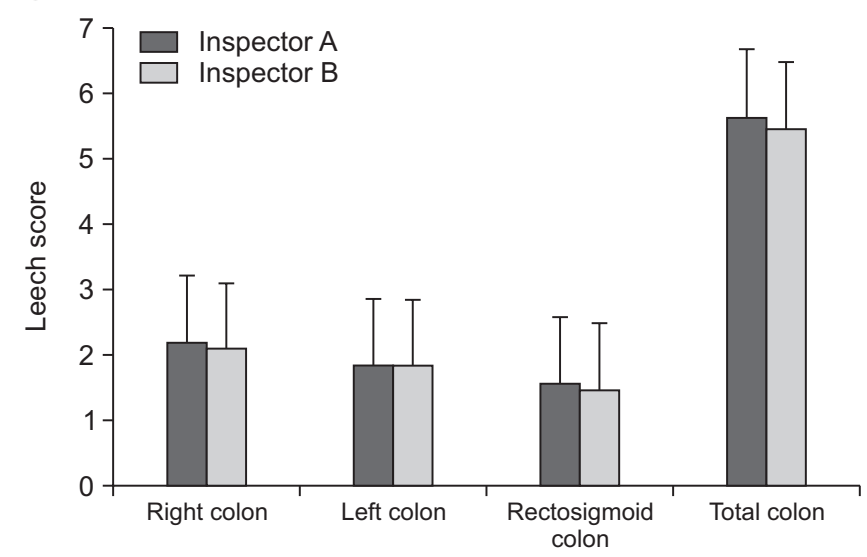

Fig. 4. Scatter diagrams of the correlation between the total colon transit time (CTT) and Starreveld score, between the total CTT and Leech score. There was statistically significant correlation between each method $(r=0.555, r=0.564)$.

Correlation between colon transit time and bowel pattern

While the total CTT showed a statistically significant positive correlation with the constipation score $(\mathrm{p}<0.05)$, there was no significant correlation between CTT and the Bristol stool form scale ( $\mathrm{p}>0.05)$.

\section{Correlation between stool retention score and bowel pattern}

Stool retention score showed a statistically significant positive correlation with the bowel pattern $(\mathrm{p}<0.05)$. However, there was no significant correlation between stool retention and the Bristol stool form scale ( $p>0.05)$.

Correlation among Modified Barthel Index, Korean vision of Mini-Mental State Examination, Constipation score, and stool retention score

There was no significant ( $p>0.05$ ) correlation among Modified Barthel Index, Korean vision of Mini-Mental State examination, Constipation score, or stool retention score.

\section{DISCUSSION}

Bowel dysfunction after stroke occurs frequently. It is widely known that there are many factors responsible for bowel dysfunction, such as neurogenic bowel, dysphagia, decreasing activities due to motor weakness, aging, inappropriate water or nutritional intake, depression, various drug intakes, and other factors $[14,15]$. However, relative- ly few studies have been performed on bowel dysfunction after stroke. Bowel dysfunction after stroke, a chronic complication due to neurogenic bowel, is difficult to manage. Additionally, bowel dysfunction complications tend to be less severe and less fatal compared to neurogenic bladder [16]. However, if there is no appropriate management for bowel dysfunction after stroke, it will limit social activities, decrease the quality of life, cause psychological and social retreat, thus making it difficult for patients to perform daily duties. It is important to have a correct evaluation to properly manage bowel dysfunction. In order to achieve this, different methods have been developed. CTT is considered an objective method for the evaluation of colonic motility [17]. CTT was used in this study as comparative criteria for different evaluation methods of bowel dysfunction. The easiest and most widely used method is clinical assessment of the bowel pattern usually conducted by taking history. Assessment on bowel pattern by history taking depends on subjective symptom and reports of patients. However, stroke patients' memories on their bowel habits are sometimes inaccurate [4], resulting in patient's subjective interpretation. CTT, a proven objective evaluation method, has a significant relationship with the symptom of constipation. CTT not only can evaluate the total colon, but also can evaluate the segmental colon [17]. However, this method is inapplicable to those who have difficulties in swallowing capsules that contain radio-opaque markers. CTT has the disadvantages of requiring at least 4 days to process, including two time plain abdominal radiography 
for the evaluation of results. Additionally, it has the limitation in outpatient clinics because patients have to intake radio-opaque Kolomark every day at the same time to have plain abdominal radiography on the 4th day at a hospital. Another objective evaluation method of constipation is plain abdominal radiography. Recently, some studies [18-20] have reported that plain abdominal radiography has poor diagnostic accuracy for constipation. However, previous studies $[7,8]$ have reported that plain abdominal radiography is significantly correlated with symptoms of constipation. Nonetheless, plain abdominal radiography does have some benefits. Compared to CTT, the plain abdominal radiography requires only onetime radiography. In addition, plain abdominal radiography has the advantages of less cost, simple to perform, and less exposure to radiation. In 2013, Park et al. [21] found that plain abdominal radiography for spinal cord injured patients had a significant correlation with CTT on constipation scores. They also reported that the interinvestigator consistency was high. Thus, plain abdominal radiography is easy to use and applicable for clinics. With the constipation score obtained through the survey of the patients with stroke, this study evaluated the degree of stool retention using the plain abdominal radiography. We also found the bowel pattern using Bristol stool form scale. We examined the correlations by evaluating CTT to determine which method would be useful for bowel dysfunction in plain abdominal radiography. We further reported the convenience in clinical application. Our results revealed that there were significant differences in constipation score and Bristol stool form scale between the constipation and the non-constipation groups. Delay of total and left colon CTT were observed in constipation group, which was consistent with previous studies $[5,6]$. Regarding the rectosigmoid colon CTT, the delay was observed in the constipation group, which was different from previous studies. With respect to the stool retention score through the plain abdominal radiography, the Starreveld score showed a statistically significant high score in the ascending colon, transverse colon, and descending colon in comparison between the constipation and the non-constipation groups, while Leech score showed a high score in the right and left colon. These results showed that there was a significant difference between the two groups with respect to the constipation score, Bristol stool form scale, total CTT, and stool retention scores. The application of Roman II Diagnosis Criteria is considered applicable to the constipation diagnosis for stroke patients. Through total CTT and plain abdominal radiography, there was a statistically significant correlation between the Starreveld score and the Leech score. Similarly, there was a statistically significant correlation between segmental CTT and segmental stool retention score. Based on these results, not only the total CTT, but also each segmental CTT could be inferred through plain abdominal radiography. Thus, in addition to CTT, plain abdominal radiography was useful as an evaluation method for bowel dysfunction in stroke patients. Furthermore, there was a statistically significant correlation in stool retention score through the plain abdominal radiography with patients' subjective symptom and constipation score reflected by the bowel pattern. Recent studies [18-20] have reported less diagnostic accuracy of plain abdominal radiography because it fails to reflect the serial bowel state of constipation. Although the amount of stool loading is subject to daily variation, our studies revealed significant correlation between CTT and plain abdominal radiography. Independently, plain abdominal radiography isn't a sufficient method to completely replace CTT. However, it can assist CTT in the diagnosis of constipation. With this correlation, we conclude that plain abdominal radiography can effectively evaluate the severity of constipation and determine the need for treatment. Because the daily performance abilities of stroke patients are significantly lower than those of normal people, examination convenience and simplicity must be considered in determining the method of choice. For patients who are treated at the outpatient clinics, it is difficult to evaluate CTT due to problems associated with its examination method and time. Therefore, to evaluate bowel dysfunction in outpatient clinics, it is necessary to prepare questionnaires to evaluate the management and conditions for the bowel dysfunction. If plain abdominal radiography is employed in replacement of the complicated CTT, the total and segmental colon activities can be known. Therefore, plain abdominal radiography is considered effective to obtain immediate results on the day of outpatient clinic visits, with the advantages of faster management and treatment as well as convenience for the patients.

Considering the characteristics of stool retention through colon segmentation on plain abdominal radiogra- 
phy, osmotic laxatives, a variety of enemas, suppositories, digital disimpaction, and other induction of bowel movement and management can be applied. Differences can arise among investigators when evaluating the degree of stool retention in plain abdominal radiography. This study employed two skilled and well-trained radiologists who understood the evaluation method well. They examined the plain abdominal radiography. The variations in results among those investigators were evaluated. There was a significant $(\mathrm{p}<0.01)$ consistency of inter-investigators regarding the degree of stool retention on using the Starreveld score and Leech score. If investigators are well trained in examining the degree of stool retention using the evaluation criteria, plain abdominal radiography can be effectively used as an objective evaluation method. However, regarding the Starreveld score which divides the total colon into four sections and evaluates each colon, there may be an overlap between the transverse and ascending colon or between the transverse and descending colon if there is excess stool retention. It may result in an error in evaluation. In such cases, it is useful to adopt the Leech score that divides the total colon into three different sections.

This study has some limitations. It did not consider patients' diets, underlying disease, or medications that might affect bowel dysfunction. Drugs that the patients previously used to control bowel dysfunction were not evaluated in this study. The correlations by comparing the evaluation methods for normal people and stroke patients were not determined. This study excluded patients who were unable to swallow Kolomark. Therefore, further studies are needed to address the above limitations.

In conclusion, this study examined the usefulness of plain abdominal radiography in evaluating bowel dysfunction after stroke. There were statistically significant correlations between the CTT and Starreveld and Leech scores evaluated by plain abdominal radiography, the most objective evaluation method for inspecting colon activities. Although plain abdominal radiography has poor diagnostic accuracy for constipation, it is useful for the evaluation of bowel dysfunction after stroke, therefore aiding the management of bowel status and implementation of appropriate treatment procedures. Due to its fast evaluation time and reduced radiation exposure, plain abdominal radiography could assist the diagnosis of constipation with applicability in clinics because of its convenience.

\section{CONFLICT OF INTEREST}

No potential conflict of interest relevant to this article was reported.

\section{ACKNOWLEDGMENTS}

This paper was supported by Wonkwang Institute of Clinical Medicine in 2014.

\section{REFERENCES}

1. Scivoletto G, Fuoco U, Badiali D, Braccl F, Lucente LD, Patrelli A, et al. Gastrointestinal dysfunction following stroke. J Neurol Sci 1997;150:S151.

2. Robain G, Chennevelle JM, Petit F, Piera JB. Incidence of constipation after recent vascular hemiplegia: a prospective cohort of 152 patients. Rev Neurol (Paris) 2002;158(5 Pt 1):589-92.

3. Harari D, Norton C, Lockwood L, Swift C. Treatment of constipation and fecal incontinence in stroke patients: randomized controlled trial. Stroke 2004;35: 2549-55.

4. Ashraf W, Park F, Lof J, Quigley EM. An examination of the reliability of reported stool frequency in the diagnosis of idiopathic constipation. Am J Gastroenterol 1996;91:26-32.

5. Yi JH, Chun MH, Kim BR, Han EY, Park JY. Bowel function in acute stroke patients. Ann Rehabil Med 2011; 35:337-43.

6. Lim YH, Kim DH, Lee MY, Joo MC. Bowel dysfunction and colon transit time in brain-injured patients. Ann Rehabil Med 2012;36:371-8.

7. Leech SC, McHugh K, Sullivan PB. Evaluation of a method of assessing faecal loading on plain abdominal radiographs in children. Pediatr Radiol 1999; 29:255-8.

8. Starreveld JS, Pols MA, Van Wijk HJ, Bogaard JW, Poen $\mathrm{H}$, Smout AJ. The plain abdominal radiograph in the assessment of constipation. Z Gastroenterol 1990; 28:335-8.

9. Thompson WG, Longstreth GF, Drossman DA, Heaton KW, Irvine EJ, Muller-Lissner SA. Functional bowel disorders and functional abdominal pain. Gut 1999;45 
Suppl 2:II43-7.

10. Cheon JH, Yoon IJ, Myung SJ, Byeon JS, Ko JE, Jung $\mathrm{KW}$, et al. Use of constipation remedies not prescribed by physician: a study of patients at a constipation clinic. J Neurogastroenterol Motil 2007;13:45-52.

11. O'Donnell LJ, Virjee J, Heaton KW. Detection of pseudodiarrhoea by simple clinical assessment of intestinal transit rate. BMJ 1990;300:439-40.

12. Arhan P, Devroede G, Jehannin B, Lanza M, Faverdin C, Dornic C, et al. Segmental colonic transit time. Dis Colon Rectum 1981;24:625-9.

13. Suh SW, Park HJ, Jung HY, Rhee JC, Rhee PL, Kim JJ, et al. Comparison of 4- day 7-day methods in the evaluation of colon transit time. Korean J Gastroenterol 2001; 38:241-6.

14. Roth EJ. Medical complications encountered in stroke rehabilitation. Phys Med Rehabil Clin North Am 1991; 2:563-77.

15. Wrenn K. Fecal impaction. N Engl J Med 1989;321:65862.

16. Seo JH, Song KS, Ko MH, Park SH. the change of neurogenic bowel dysfunction in spinal cord injury pa- tients during admission. J Korean Acad Rehabil Med 2009;33:441-7.

17. Keighley MR, Henry MM, Bartolo DC, Mortensen NJ. Anorectal physiology measurement: report of a working party. Br J Surg 1989;76:356-7.

18. Reuchlin-Vroklage LM, Bierma-Zeinstra S, Benninga MA, Berger MY. Diagnostic value of abdominal radiography in constipated children: a systematic review. Arch Pediatr Adolesc Med 2005;159:671-8.

19. Pensabene L, Buonomo C, Fishman L, Chitkara D, Nurko S. Lack of utility of abdominal x-rays in the evaluation of children with constipation: comparison of different scoring methods. J Pediatr Gastroenterol Nutr 2010;51:155-9.

20. Moylan S, Armstrong J, Diaz-Saldano D, Saker M, Yerkes EB, Lindgren BW. Are abdominal x-rays a reliable way to assess for constipation? J Urol 2010;184(4 Suppl):1692-8.

21. Park HJ, Noh SE, Kim GD, Joo MC. Plain abdominal radiograph as an evaluation method of bowel dysfunction in patients with spinal cord injury. Ann Rehabil Med 2013;37:547-55. 